\title{
TOPOLOGICAL STRUCTURE \\ OF THE SOLUTION SET OF SINGULAR EQUATIONS \\ WITH SIGN CHANGING TERMS UNDER DIRICHLET BOUNDARY CONDITION
}

José V. Gonçalves - Marcos R. Marcial - Olimpio H. Miyagaki

ABSTRACT. In this paper we establish existence of connected components of positive solutions of the equation $-\Delta_{p} u=\lambda f(u)$ in $\Omega$, under Dirichlet boundary conditions, where $\Omega \subset \mathbb{R}^{N}$ is a bounded domain with smooth boundary $\partial \Omega, \Delta_{p}$ is the $p$-Laplacian, and $f:(0, \infty) \rightarrow \mathbb{R}$ is a continuous function which may blow up to $\pm \infty$ at the origin.

\section{Introduction}

In this paper we establish existence of a continuum of positive solutions of

$(\mathrm{P})_{\lambda}$

$$
\begin{cases}-\Delta_{p} u=\lambda f(u) & \text { in } \Omega, \\ u>0 & \text { in } \Omega, \\ u=0 & \text { on } \partial \Omega,\end{cases}
$$

where $\Omega \subset \mathbb{R}^{N}$ is a bounded domain with smooth boundary $\partial \Omega, \Delta_{p}$ is the $p$ Laplacian, $1<p<\infty, \lambda>0$ is a real parameter, $f:(0, \infty) \rightarrow \mathbb{R}$ is a continuous function which may blow up to $\pm \infty$ at the origin.

2010 Mathematics Subject Classification. 35J25, 35J55, 35J70.

Key words and phrases. Connected sets; fixed points; Schauder theory; elliptic equations.

This work was supported by CNPq/CAPES/PROCAD/UFG/UnB-Brazil

The first and the third authors were supported in part by $\mathrm{CNPq} / \mathrm{Brazil}$.

The second author was supported by CAPES/Brazil. 
Definition 1.1. By a solution of $(\mathrm{P})_{\lambda}$ we mean a function $u \in W_{0}^{1, p}(\Omega) \cap$ $C(\bar{\Omega})$, with $u>0$ in $\Omega$, such that

$$
\int_{\Omega}|\nabla u|^{p-2} \nabla u \cdot \nabla \varphi d x=\lambda \int_{\Omega} f(u) \varphi d x, \quad \varphi \in W_{0}^{1, p}(\Omega) .
$$

Definition 1.2. The solution set of $(\mathrm{P})_{\lambda}$ is

$$
\mathcal{S}:=\left\{(\lambda, u) \in(0, \infty) \times C(\bar{\Omega}) \mid u \text { is a solution of }(\mathrm{P})_{\lambda}\right\}
$$

In the pioneering work [5], Crandall, Rabinowitz and Tartar employed topological methods, Schauder Theory, and Maximum Principles to prove existence of an unbounded connected subset in $\mathbb{R} \times C_{0}(\bar{\Omega})$ of positive solutions $u \in$ $C^{2}(\Omega) \cap C(\bar{\Omega})$ of the problem

$$
\begin{cases}-L u=g(x, u) & \text { in } \Omega, \\ u=0 & \text { on } \partial \Omega,\end{cases}
$$

where $L$ is a linear second order uniformly elliptic operator,

$$
C_{0}(\bar{\Omega})=\{u \in C(\bar{\Omega}) \mid u=0 \text { on } \partial \Omega\}
$$

and $g: \bar{\Omega} \times(0, \infty) \rightarrow(0, \infty)$ is a continuous function satisfying $g(x, t) \stackrel{t \rightarrow 0^{+}}{\longrightarrow} 0$ uniformly for $x \in \bar{\Omega}$. A typical example is $g(x, t)=t^{\gamma}$, where $\gamma>0$.

Several techniques have been employed in the study of $\left(\mathrm{P}_{\lambda}\right)$. In [11], Giacomoni, Schindler and Takac employed variational methods to investigate the problem

$$
\begin{cases}-\Delta_{p} u=\frac{\lambda}{u^{\delta}}+u^{q} & \text { in } \Omega, \\ u>0 & \text { in } \Omega, \\ u=0 & \text { on } \partial \Omega,\end{cases}
$$

where $1<p<\infty, p-1<q<p^{*}-1, \lambda>0$ and $0<\delta<1$ with $p^{*}=N p /(N-p)$ if $1<p<N, p^{*} \in(N, \infty)$ if $p=N$, and $p^{*}=\infty$ if $p>N$. Several results were shown in that paper, among them existence, multiplicity and regularity of solutions.

In the present work we exploit the topological structure of the solution set of $\left(\mathrm{P}_{\lambda}\right)$ and our main assumptions are:

$\left(\mathrm{f}_{1}\right) f:(0, \infty) \rightarrow \mathbb{R}$ is continuous and

$$
\lim _{u \rightarrow \infty} \frac{f(u)}{u^{p-1}}=0,
$$

$\left(\mathrm{f}_{2}\right)$ there are positive numbers $a, \beta, A$ with $\beta<1$ such that

(i) $f(u) \geq a / u^{\beta}$ for $u>A$,

(ii) $\limsup u^{\beta}|f(u)|<\infty$.

The main result of this paper is: 
Theorem 1.3. Assume $\left(\mathrm{f}_{1}\right)-\left(\mathrm{f}_{2}\right)$. Then there is a number $\lambda_{0}>0$ and a connected subset $\Sigma$ of $\left[\lambda_{0}, \infty\right) \times C(\bar{\Omega})$ satisfying

$$
\begin{gathered}
\Sigma \subset \mathcal{S}, \\
\Sigma \cap(\{\lambda\} \times C(\bar{\Omega})) \neq \emptyset, \quad \lambda_{0} \leq \lambda<\infty .
\end{gathered}
$$

There is a broad literature on singular problems and we further refer the reader to Lazer and McKenna in [16], Diaz, Morel and Oswald [8], Gerghu and Radulescu [10], Goncalves, Rezende and Santos [13], Hai [14, 15], Mohammed [19], Shi and Yao [21], Hoang Loc and Schmitt [18], Carl and Perera [4], and their references.

Our result includes examples such as

$$
\begin{aligned}
& u^{q}-\frac{1}{u^{\beta}}, \quad \beta>0,0<q<p-1, \\
& \frac{1}{u^{\beta}}-\frac{1}{u^{\alpha}}, \quad 0<\beta<\alpha<1, \\
& \ln (u) .
\end{aligned}
$$

In the proof of our Theorem 1.3 we shall employ topological arguments to construct a suitable connected component of the solution set $\mathcal{S}$ of $(\mathrm{P})_{\lambda}$. More precisely, we shall use in a nontrivial way Theorem 2.1 from Sun and Song [23] whose proof is based on the famous lemma of Whyburn, (cf. [26, Theorem 9.3]). At first some notations:

Let $M=(M, d)$ be a metric space and denote by $\left\{\Sigma_{n}\right\}$ a sequence of connected components of $M$. The upper limit of $\left\{\Sigma_{n}\right\}$ is defined by

$$
\varlimsup \Sigma_{n}=\left\{u \in M \mid \text { there is }\left(u_{n_{i}}\right) \subseteq \bigcup \Sigma_{n} \text { with } u_{n_{i}} \in \Sigma_{n_{i}} \text { and } u_{n_{i}} \rightarrow u\right\} .
$$

REMARK 1.4. $\varlimsup \Sigma_{n}$ is a closed subset of $M$.

TheOREm 1.5. Let $M$ be a metric space and $\left\{\alpha_{n}\right\},\left\{\beta_{n}\right\} \in \mathbb{R}$ be sequences satisfying $\ldots<\alpha_{n}<\ldots<\alpha_{1}<\beta_{1}<\ldots<\beta_{n}<\ldots$ with $\alpha_{n} \rightarrow-\infty$ and $\beta_{n} \rightarrow \infty$. Assume that $\left\{\Sigma_{n}^{*}\right\}$ is a sequence of connected subsets of $\mathbb{R} \times M$ satisfying:

(a) $\Sigma_{n}^{*} \cap\left(\left\{\alpha_{n}\right\} \times M\right) \neq \emptyset$,

(b) $\Sigma_{n}^{*} \cap\left(\left\{\beta_{n}\right\} \times M\right) \neq \emptyset$,

for each $n$. For each $\alpha, \beta \in(-\infty, \infty)$ with $\alpha<\beta$,

(c) $\left(\bigcup \Sigma_{n}^{*}\right) \cap([\alpha, \beta] \times M)$ is a relatively compact subset of $\mathbb{R} \times M$.

Then there is a number $\lambda_{0}>0$ and a connected component $\Sigma^{*}$ of $\overline{\lim } \Sigma_{n}^{*}$ such that $\Sigma^{*} \cap(\{\lambda\} \times M) \neq \emptyset$ for each $\lambda \in\left(\lambda_{0}, \infty\right)$. 


\section{Some auxiliary results}

We gather below a few technical results. For completeness, a few proofs will be provided in the appendix. The Euclidean distance from $x \in \Omega$ to $\partial \Omega$ is

$$
d(x)=\operatorname{dist}(x, \partial \Omega) .
$$

The result below derives from Gilbarg and Trudinger [12], and Vàzquez [25].

Lemma 2.1. Let $\Omega \subset \mathbb{R}^{N}$ be a smooth bounded domain. Then:

(a) $d \in \operatorname{Lip}(\bar{\Omega})$ and $d$ is $C^{2}$ in a neighbourhood of $\partial \Omega$,

(b) if $\phi_{1}$ denotes a positive eigenfunction of $\left(-\Delta_{p}, W_{0}^{1, p}(\Omega)\right)$ one has

$$
\phi_{1} \in C^{1, \alpha}(\bar{\Omega}) \quad \text { with } 0<\alpha<1, \quad \frac{\partial \phi_{1}}{\partial \nu}<0 \quad \text { on } \partial \Omega,
$$

and there are positive constants $C_{1}, C_{2}$ such that

$$
C_{1} d(x) \leq \phi_{1}(x) \leq C_{2} d(x), \quad x \in \Omega .
$$

The result below is due to Crandall, Rabinowitz and Tartar [5], Lazer and McKenna [16] in the case $p=2$ and Giacomoni, Schindler and Takac [11] in the case $1<p<\infty$.

Lemma 2.2. Let $\beta \in(0,1)$ and $m>0$. Then the problem

$$
\begin{cases}-\Delta_{p} u=\frac{m}{u^{\beta}} & \text { in } \Omega, \\ u>0 & \text { in } \Omega, \\ u=0 & \text { on } \partial \Omega,\end{cases}
$$

admits only a weak solution $u_{m} \in W_{0}^{1, p}(\Omega)$. Moreover, $u_{m} \geq \varepsilon_{m} \phi_{1}$ in $\Omega$ for some constant $\varepsilon_{m}>0$.

REMARK 2.3. By the results in [17], [11], there is $\alpha \in(0,1)$ such that $u_{m} \in$ $C^{1, \alpha}(\bar{\Omega})$.

The result below, which is crucial in this work, and whose proof is provided in the appendix, is basically due to Hai [15].

Lemma 2.4. Let $g \in L_{\mathrm{loc}}^{\infty}(\Omega)$. Assume that there is $\beta \in(0,1)$ and $C>0$ such that

$$
|g(x)| \leq \frac{C}{d(x)^{\beta}}, \quad x \in \Omega .
$$

Then there is only a weak solution $u \in W_{0}^{1, p}(\Omega)$ of

$$
\begin{cases}-\Delta_{p} u=g & \text { in } \Omega, \\ u=0 & \text { on } \partial \Omega .\end{cases}
$$

In addition, there exist constants $\alpha \in(0,1)$ and $M>0$, with $M$ depending only on $C, \beta, \Omega$ such that $u \in C^{1, \alpha}(\bar{\Omega})$ and $\|u\|_{C^{1, \alpha}(\bar{\Omega})} \leq M$. 
REMARK 2.5. The solution operator associated to (2.3) is: let

$$
\begin{gathered}
\mathcal{M}_{\beta, \infty}=\left\{g \in L_{l o c}^{\infty}(\Omega)|| g(x) \mid \leq \frac{C}{d(x)^{\beta}}, x \in \Omega\right\}, \\
S: \mathcal{M}_{\beta, \infty} \rightarrow W_{0}^{1, p}(\Omega) \cap C^{1, \alpha}(\bar{\Omega}), \quad S(g):=u .
\end{gathered}
$$

Notice that $\|S(g)\|_{C^{1, \alpha}(\bar{\Omega})} \leq M$, for all $g \in \mathcal{M}_{C, d, \beta, \infty}$, with $M$ depending only on $C, \beta, \Omega$.

Corollary 2.6. Let $g, \widetilde{g} \in L_{\mathrm{loc}}^{\infty}(\Omega)$ with $g \geq 0, g \neq 0$ satisfying (2.2). Then, for each $\varepsilon>0$, the problem

$$
\begin{cases}-\Delta_{p} u_{\varepsilon}=g \chi_{\{d>\varepsilon\}}+\widetilde{g} \chi_{\{d<\varepsilon\}} & \text { in } \Omega \\ u_{\epsilon}=0 & \text { on } \partial \Omega\end{cases}
$$

admits only a solution $u_{\varepsilon} \in C^{1, \alpha}(\bar{\Omega})$ for some $\alpha \in(0,1)$. In addition, there is $\varepsilon_{0}>0$ such that

$$
u_{\varepsilon} \geq \frac{u}{2} \quad \text { in } \Omega \text { for each } \varepsilon \in\left(0, \varepsilon_{0}\right),
$$

where $u$ is the solution of (2.3).

A proof of the corollary above will be included in the appendix.

\section{Lower and upper solutions}

In this section we present two results, due to Hai [15, Theorem 2.1], on existence of lower and upper solutions of $(\mathrm{P})_{\lambda}$. At first some definitions.

Definition 3.1. A function $\underline{u} \in W_{0}^{1, p}(\Omega)$ with $\underline{u}>0$ in $\Omega$ such that

$$
\int_{\Omega}|\nabla \underline{u}|^{p-2} \nabla \underline{u} \cdot \nabla \varphi d x \leq \lambda \int_{\Omega} f(\underline{u}) \varphi d x, \quad \varphi \in W_{0}^{1, p}(\Omega), \varphi \geq 0,
$$

is a lower solution of $(\mathrm{P})_{\lambda}$.

Definition 3.2. A function $\bar{u} \in W_{0}^{1, p}(\Omega)$ with $\bar{u}>0$ in $\Omega$ such that

$$
\int_{\Omega}|\nabla \bar{u}|^{p-2} \nabla \bar{u} \cdot \nabla \varphi d x \geq \lambda \int_{\Omega} f(\bar{u}) \varphi d x, \quad \varphi \in W_{0}^{1, p}(\Omega), \varphi \geq 0,
$$

is an upper solution of $(\mathrm{P})_{\lambda}$.

We establish the existence of a lower solution.

Theorem 3.3. Assume $\left(\mathrm{f}_{1}\right)-\left(\mathrm{f}_{2}\right)$. Then there exist $\lambda_{0}>0$ and a non-negative function $\psi \in C^{1, \alpha}(\bar{\Omega})$, with $\psi>0$ in $\Omega, \psi=0$ on $\partial \Omega, \alpha \in(0,1)$ such that for each $\lambda \in\left[\lambda_{0}, \infty\right), \underline{u}=\lambda^{r} \psi$ with $r=1 /(p+\beta-1)$, is a lower solution of $(\mathrm{P})_{\lambda}$.

Proof of Theorem 3.3. See Hai [15, p. 622]. 
By Lemma 2.2, there are both a function $\phi \in C^{1, \alpha}(\bar{\Omega})$, with $\alpha \in(0,1)$, such that

$$
\begin{cases}-\Delta_{p} \phi=\frac{1}{\phi^{\beta}} & \text { in } \Omega, \\ \phi>0 & \text { in } \Omega, \\ \phi=0 & \text { on } \partial \Omega,\end{cases}
$$

and a constant $C_{1}>0$ such that $\phi \geq C_{1} d$ in $\Omega$.

Next, we establish the existence of an upper solution.

Theorem 3.4. Assume $\left(\mathrm{f}_{1}\right)-\left(\mathrm{f}_{2}\right)$ and take $\Lambda>\lambda_{0}$ with $\lambda_{0}$ as in Theorem 3.3 . Then for each $\lambda \in\left[\lambda_{0}, \Lambda\right],(\mathrm{P})_{\lambda}$ admits an upper solution $\bar{u}=\bar{u}_{\lambda}=M \phi$ where $M>0$ is a constant and $\phi$ is given by (3.1).

Proof of Theorem 3.4. See Hai in [15, p. 623].

\section{Further technical results}

At first we introduce some notations, remarks and lemmas. Take $\Lambda>\lambda_{0}$ and set $I_{\Lambda}:=\left[\lambda_{0}, \Lambda\right]$. For each $\lambda \in I_{\Lambda}$, by Theorem 3.3,

$$
\underline{u}=\underline{u}_{\lambda}=\lambda^{r} \psi
$$

is a lower solution of $(\mathrm{P})_{\lambda}$. Pick $M=M_{\Lambda} \geq \Lambda^{r} \delta^{1 /(p-1)}$. By Theorem 3.4,

$$
\bar{u}=\bar{u}_{\lambda}=M_{\Lambda} \phi
$$

is an upper solution of $(\mathrm{P})_{\lambda}$. It follows that

$$
\underline{u}=\lambda^{r} \psi \leq \Lambda^{r} \delta^{1 /(p-1)} \phi \leq M \phi=\bar{u} .
$$

The convex, closed subset of $I_{\Lambda} \times C(\bar{\Omega})$, defined by

$$
\mathcal{G}_{\Lambda}:=\left\{(\lambda, u) \in I_{\Lambda} \times C(\bar{\Omega}) \mid \lambda \in I_{\Lambda}, \underline{u} \leq u \leq \bar{u} \text { and } u=0 \text { on } \partial \Omega\right\}
$$

will play a key role in this work.

For each $u \in C(\bar{\Omega})$ define

$$
f_{\Lambda}(u)=\chi_{S_{1}} f(\underline{u})+\chi_{S_{2}} f(u)+\chi_{S_{3}} f(\bar{u}), \quad x \in \Omega,
$$

where $S_{1}:=\{x \in \Omega \mid u(x)<\underline{u}(x)\}, S_{2}:=\{x \in \Omega \mid \underline{u}(x) \leq u(x) \leq \bar{u}(x)\}$, $S_{3}:=\{x \in \Omega \mid \bar{u}(x)<u(x)\}$, and $\chi_{S_{i}}$ is the characteristic function of $S_{i}$.

Lemma 4.1. For each $u \in C(\bar{\Omega}), f_{\Lambda}(u) \in L_{\mathrm{loc}}^{\infty}(\Omega)$ and there are $C>0$, $\beta \in(0,1)$ such that

$$
\left|f_{\Lambda}(u)(x)\right| \leq \frac{C}{d(x)^{\beta}}, \quad x \in \Omega
$$


Proof. Indeed, let $\mathcal{K} \subset \Omega$ be a compact subset. Then both $\underline{u}$ and $\bar{u}$ achieve a positive maximum and a positive minimum on $\mathcal{K}$. Since $f$ is continuous in $(0, \infty)$ then $f_{\Lambda}(u) \in L_{\mathrm{loc}}^{\infty}(\Omega)$.

Verification of (4.3): Since $\Omega=\bigcup_{i=1}^{3} S_{i}$ it is enough to show that

$$
|f(u(x))| \leq \frac{C}{d(x)^{\beta}}, \quad x \in S_{i}, \quad i=1,2,3 .
$$

At first, by $\left(\mathrm{f}_{2}\right)(\mathrm{ii})$ there are $C, \delta>0$ such that

$$
|f(s)| \leq \frac{C}{s^{\beta}}, \quad 0<s<\delta .
$$

Let $\Omega_{\delta}=\{x \in \Omega \mid d(x)<\delta\}$. Recalling that $\underline{u} \in C^{1}(\bar{\Omega})$, let

$$
D=\max _{\bar{\Omega}} d(x), \quad \nu_{\delta}:=\min _{\overline{\Omega_{\delta}^{c}}} d(x), \quad \nu^{\delta}:=\max _{\overline{\Omega_{\delta}^{c}}} d(x),
$$

and notice that both $0<\nu_{\delta} \leq \nu^{\delta} \leq D<\infty$ and $f\left(\left[\nu_{\delta}, \nu^{\delta}\right]\right)$ are compact.

On the other hand, applying Theorems 3.3, 3.4, Lemmas 2.1 and 2.2 we infer that $0<\lambda_{0}^{r} \psi \leq \lambda^{r} \psi=\underline{u} \leq \bar{u}=M \phi$ in $\Omega$ and

$$
\frac{1}{\underline{u}^{\beta}}, \frac{1}{\bar{u}^{\beta}} \leq \frac{1}{\left(\lambda_{0}^{r} \psi(x)\right)^{\beta}} \leq \frac{C}{d(x)^{\beta}}, \quad x \in \Omega_{\delta} .
$$

To finish the proof, we distinguish three cases:

(1) $x \in S_{1}$. In this case, $f_{\Lambda}(u(x))=f(\underline{u}(x))$. If $x \in S_{1} \cap \Omega_{\delta}$ we infer that

$$
\left|f_{\Lambda}(u(x))\right| \leq \frac{C}{\underline{u}(x)^{\beta}} \leq \frac{C}{d(x)^{\beta}} .
$$

If $x \in S_{1} \cap \Omega_{\delta}^{c}$ pick positive numbers $d_{i}, i=1,2$, such that $d_{1} \leq \underline{u}(x) \leq d_{2}$, $x \in \Omega_{\delta}^{c}$. Hence

$$
\left|f_{\Lambda}(u(x))\right| \leq \frac{C}{d(x)^{\beta}}, \quad x \in \Omega .
$$

(2) $x \in S_{2}$. In this case, $0<\lambda_{0}^{r} \psi \leq u \leq M \phi$ and, as a consequence,

$$
|f(u(x))| \leq \frac{C}{u(x)^{\beta}}, \quad x \in \Omega_{\delta}
$$

Hence, there is a positive constant $\widetilde{C}$ such that $|f(u(x))| \leq \widetilde{C}, x \in \overline{\Omega_{\delta}^{c}}$. Thus

$$
|f(u(x))| \leq \begin{cases}\widetilde{C} & \text { if } x \in \overline{\Omega_{\delta}^{c}} \\ \frac{C}{d(x)^{\beta}} & \text { if } x \in \Omega_{\delta}\end{cases}
$$

On the other hand,

$$
\frac{1}{D^{\beta}} \leq \frac{1}{d(x)^{\beta}}, \quad x \in \overline{\Omega_{\delta}^{c}}
$$


and therefore there is a constant $C>0$ such that

$$
|f(u(x))| \leq \begin{cases}\frac{C}{D^{\beta}} & \text { if } x \in \overline{\Omega_{\delta}^{c}}, \\ \frac{C}{d(x)^{\beta}} & \text { if } x \in \Omega_{\delta} .\end{cases}
$$

Therefore,

$$
|f(u(x))| \leq \frac{C}{d(x)^{\beta}}, \quad x \in S_{2}, u \in \mathcal{G}_{\Lambda} .
$$

(3) $x \in S_{3}$. In this case $f_{\Lambda}(u(x))=f(\bar{u}(x))$. If $x \in S_{3} \cap \Omega_{\delta}$ we infer that

$$
\left|f_{\Lambda}(u(x))\right| \leq \frac{C}{\bar{u}(x)^{\beta}} \leq \frac{C}{d(x)^{\beta}} .
$$

If $x \in S_{3} \cap \Omega_{\delta}^{c}$. Pick positive numbers $d_{i}, i=1,2$, such that $\left.d_{1} \leq \bar{u}(x)\right) \leq d_{2}$, $x \in \Omega_{\delta}^{c}$. Hence

$$
\left|f_{\Lambda}(u(x))\right| \leq \frac{C}{d(x)^{\beta}}, \quad x \in \Omega .
$$

This ends the proof of Lemma 4.1.

Remark 4.2. By Lemmas 2.4,4.1 and Remark 2.5, for each $v \in C(\overline{\Omega)}$ and $\lambda \in I_{\Lambda}$,

$$
\lambda f_{\Lambda}(v) \in L_{\mathrm{loc}}^{\infty}(\Omega) \quad \text { and } \quad\left|\lambda f_{\Lambda}(v)\right| \leq \frac{C_{\Lambda}}{d^{\beta}(x)} \quad \text { in } \Omega,
$$

where $C_{\Lambda}>0$ is a constant independent of $v$ and $\beta \in(0,1)$. So for each $v$,

$$
\begin{cases}-\Delta_{p} u=\lambda f_{\Lambda}(v) & \text { in } \Omega, \\ u=0 & \text { on } \partial \Omega,\end{cases}
$$

admits only a solution $u=S\left(\lambda f_{\Lambda}(v)\right) \in W_{0}^{1, p}(\Omega) \cap C^{1, \alpha}(\bar{\Omega})$.

Set $F_{\Lambda}(u)(x)=f_{\Lambda}(u(x)), u \in C(\bar{\Omega})$, and consider the operator

$$
\begin{gathered}
T: I_{\Lambda} \times C(\bar{\Omega}) \rightarrow W_{0}^{1, p}(\Omega) \cap C^{1, \alpha}(\bar{\Omega}), \\
T(\lambda, u)=S\left(\lambda F_{\Lambda}(u)\right) \quad \text { if } \lambda_{0} \leq \lambda \leq \Lambda, u \in C(\bar{\Omega}) .
\end{gathered}
$$

Notice that if $(\lambda, u) \in I_{\Lambda} \times C(\bar{\Omega})$ satisfies $u=T(\lambda, u)$ then $u$ is a solution of

$$
\begin{cases}-\Delta_{p} u=\lambda f_{\Lambda}(u) & \text { in } \Omega, \\ u=0 & \text { on } \partial \Omega .\end{cases}
$$

Lemma 4.3. If $(\lambda, u) \in I_{\Lambda} \times C(\bar{\Omega})$ and $u=T(\lambda, u)$ then $(\lambda, u) \in \mathcal{G}_{\Lambda}$.

Proof. Indeed, let $(\lambda, u) \in I_{\Lambda} \times C(\bar{\Omega})$ such that $T(\lambda, u)=u$. Then

$$
\int_{\Omega}|\nabla u|^{p-2} \nabla u . \nabla \varphi d x=\lambda \int_{\Omega} f_{\Lambda}(u) \varphi d x, \quad \varphi \in W_{0}^{1, p}(\Omega) .
$$


We claim that $u \geq \underline{u}$. Assume on the contrary, that $\varphi:=(\underline{u}-u)^{+} \not \equiv 0$. Then

$$
\begin{aligned}
\int_{\Omega}|\nabla u|^{p-2} \nabla u \cdot \nabla \varphi d x & =\int_{u<\underline{u}}|\nabla u|^{p-2} \nabla u \cdot \nabla \varphi d x \\
& =\lambda \int_{u<\underline{u}} f_{\Lambda}(u) \cdot \varphi d x=\lambda \int_{u<\underline{u}} f(\underline{u}) \cdot \varphi d x \\
& \geq \int_{u<\underline{u}}|\nabla \underline{u}|^{p-2} \nabla \underline{u} \cdot \nabla \varphi d x=\int_{\Omega}|\nabla \underline{u}|^{p-2} \nabla \underline{u} \cdot \nabla \varphi d x .
\end{aligned}
$$

Hence

$$
\int_{\Omega}\left[|\nabla u|^{p-2} \nabla u-|\nabla \underline{u}|^{p-2} \nabla \underline{u}\right] \cdot \nabla(u-\underline{u}) d x \leq 0 .
$$

It follows, by Lemma 1.2 , that $\int_{\Omega}|\nabla(u-\underline{u})|^{p} d x \leq 0$, contradicting $\varphi \not \equiv 0$. Thus, $(\underline{u}-u)^{+}=0$, that is, $\underline{u}-u \leq 0$, and so $\underline{u} \leq T(\lambda, u)$.

We claim that $\bar{u} \geq u$. Assume on the contrary that $\varphi:=(u-\bar{u})^{+} \not \equiv 0$. We have

$$
\begin{aligned}
\int_{\Omega}|\nabla u|^{p-2} \nabla u \cdot \nabla \varphi d x & =\int_{\bar{u}<u}|\nabla u|^{p-2} \nabla u \cdot \nabla \varphi d x \\
& =\lambda \int_{\bar{u}<u} f_{\Lambda}(u) \cdot \varphi d x=\lambda \int_{\bar{u}<u} f(\bar{u}) \cdot \varphi d x \\
& \leq \int_{\bar{u}<u}|\nabla \bar{u}|^{p-2} \nabla \bar{u} \cdot \nabla \varphi d x=\int_{\Omega}|\nabla \bar{u}|^{p-2} \nabla \bar{u} \cdot \nabla \varphi d x .
\end{aligned}
$$

Therefore,

$$
\int_{\Omega}\left[|\nabla u|^{p-2} \nabla u-|\nabla \bar{u}|^{p-2} \nabla \bar{u}\right] \cdot \nabla(u-\bar{u}) d x \leq 0,
$$

contradicting $\varphi \not \equiv 0$. Thus $(u-\bar{u})^{+}=0$ so that $u-\bar{u} \leq 0$, which gives $\bar{u} \geq$ $T(\lambda, u)$. As a consequence of the arguments above $u \in \mathcal{G}_{\Lambda}$, showing Lemma 4.3.

REMARK 4.4. By the definitions of $f_{\Lambda}$ and $\mathcal{G}_{\Lambda}$, for each $(\lambda, u) \in \mathcal{G}_{\Lambda}$

$$
f_{\Lambda}(u)=f(u), \quad x \in \Omega .
$$

REMARK 4.5. By Remark 2.5, there is $R_{\Lambda}>0$ such that $\mathcal{G}_{\Lambda} \subset B\left(0, R_{\Lambda}\right) \subset$ $C(\bar{\Omega})$ and

$$
T\left(I_{\Lambda} \times \overline{B\left(0, R_{\Lambda}\right)}\right) \subseteq B\left(0, R_{\Lambda}\right) .
$$

Notice that, by (4.6) and Lemma 4.3, if $(\lambda, u) \in I_{\Lambda} \times C(\bar{\Omega})$ satisfies $u=T(\lambda, u)$ then $(\lambda, u)$ is a solution of $(\mathrm{P})_{\lambda}$. By Remark 4.4, to solve $(\mathrm{P})_{\lambda}$ it suffices to look for fixed points of $T$.

Lemma 4.6. $T: I_{\Lambda} \times \overline{B\left(0, R_{\Lambda}\right)} \rightarrow \overline{B\left(0, R_{\Lambda}\right)}$ is continuous and compact.

Proof. Let $\left\{\left(\lambda_{n}, u_{n}\right)\right\} \subseteq I_{\Lambda} \times \overline{B\left(0, R_{\Lambda}\right)}$ be a sequence such that

$$
\lambda_{n} \rightarrow \lambda \quad \text { and } \quad u_{n} \stackrel{C(\bar{\Omega})}{\longrightarrow} u, \quad \text { as } n \rightarrow \infty .
$$


Set $v_{n}=T\left(\lambda_{n}, u_{n}\right)$ and $v=T(\lambda, u)$ so that $v_{n}=S\left(\lambda_{n} F_{\Lambda}\left(u_{n}\right)\right)$ and $v=$ $S\left(\lambda F_{\Lambda}(u)\right)$. It follows that

$$
\begin{aligned}
\int_{\Omega}\left(\left|\nabla v_{n}\right|^{p-2} \nabla v_{n}-|\nabla v|^{p-2} \nabla v\right) \cdot \nabla\left(v_{n}-v\right) d x & \\
& =\lambda_{n} \int_{\Omega}\left(f_{\Lambda}\left(u_{n}\right)-f_{\Lambda}(u)\right)\left(v_{n}-v\right) d x \leq C \int_{\Omega}\left|f_{\Lambda}\left(u_{n}\right)-f_{\Lambda}(u)\right| d x .
\end{aligned}
$$

Since

$$
\left|f_{\Lambda}\left(u_{n}\right)-f_{\Lambda}(u)\right| \leq \frac{C}{d(x)^{\beta}} \in L^{1}(\Omega) \quad \text { and } \quad f_{\Lambda}\left(u_{n}(x)\right) \rightarrow f_{\Lambda}(u(x)) \quad \text { a.e. } x \in \Omega,
$$

as $n \rightarrow \infty$, it follows by Lebesgue's theorem that

$$
\int_{\Omega}\left|f_{\Lambda}\left(u_{n}\right)-f_{\Lambda}(u)\right| d x \rightarrow 0, \quad \text { as } n \rightarrow \infty .
$$

Therefore $v_{n} \rightarrow v$, as $n \rightarrow \infty$ in $W_{0}^{1, p}(\Omega)$. On the other hand, since $u_{n} \stackrel{C(\bar{\Omega})}{\longrightarrow} u$, as $n \rightarrow \infty$, by the proof of Lemma 4.1,

$$
\lambda_{n} f_{\Lambda}\left(u_{n}\right) \in L_{\mathrm{loc}}^{\infty}(\Omega) \quad \text { and } \quad\left|\lambda_{n} f_{\Lambda}\left(u_{n}\right)\right| \leq \frac{C_{\Lambda}}{d^{\beta}(x)} \quad \text { in } \Omega .
$$

By Lemma 2.4, there is a constant $M>0$ such that $\left\|v_{n}\right\|_{C^{1, \alpha}(\bar{\Omega})} \leq M$ so that $v_{n} \stackrel{C(\bar{\Omega})}{\longrightarrow} v$. This shows that $T: I_{\Lambda} \times \overline{B\left(0, R_{\Lambda}\right)} \rightarrow \overline{B\left(0, R_{\Lambda}\right)}$ is continuous.

The compactness of $T$ follows from the arguments in the five lines above.

\section{Bounded connected sets of solutions of $\left(\mathrm{P}_{\lambda}\right)$}

By applying the previous technical results and the Leray-Schauder Continuation theorem (see [6]) which we state below regarding the use of its notations, we get

Theorem 5.1. Let D be an open bounded subset of the Banach space X. Let $a, b \in \mathbb{R}$ with $a<b$ and assume that $T:[a, b] \times \bar{D} \rightarrow X$ is compact and continuous. Consider $\Phi:[a, b] \times \bar{D} \rightarrow X$ defined by $\Phi(t, u)=u-T(t, u)$. Assume that

(a) $\Phi(t, u) \neq 0, t \in[a . b], u \in \partial D$,

(b) $\operatorname{deg}(\Phi(t, \cdot), D, 0) \neq 0$ for some $t \in[a, b]$,

and set $\mathcal{S}_{a, b}=\{(t, u) \in[a, b] \times \bar{D} \mid \Phi(t, u)=0\}$. Then, there is a connected compact subset $\Sigma_{a, b}$ of $\mathcal{S}_{a, b}$ such that

$$
\Sigma_{a, b} \cap(\{a\} \times D) \neq \emptyset \quad \text { and } \quad \Sigma_{a, b} \cap(\{b\} \times D) \neq \emptyset .
$$

We will be able to show the following auxiliary result.

Theorem 5.2. Assume $\left(\mathrm{f}_{1}\right)-\left(\mathrm{f}_{2}\right)$. Then there is a number $\lambda_{0}>0$ and for each $\Lambda>\lambda_{0}$ there is a connected set $\Sigma_{\Lambda} \subset\left(\left[\lambda_{0}, \Lambda\right] \times C(\bar{\Omega})\right.$ satisfying:

$$
\Sigma_{\Lambda} \subset \mathcal{S}, \quad \Sigma_{\Lambda} \cap\left(\left\{\lambda_{0}\right\} \times C(\bar{\Omega})\right) \neq \emptyset, \quad \Sigma_{\Lambda} \cap(\{\Lambda\} \times C(\bar{\Omega})) \neq \emptyset .
$$


Proof of Theorem 5.2. At first, some notations and technical results are needed. The Leray-Schauder theorem above will be applied to the operator $T$ in the settings of Section 4. Remember that $T$ is continuous, compact and $T\left(I_{\Lambda} \times \overline{B\left(0, R_{\Lambda}\right)}\right) \subset B\left(0, R_{\Lambda}\right)$.

Consider $\left.\Phi: I_{\Lambda} \times \overline{B(0, R)} \rightarrow \overline{B(0, R)}\right)$ defined by $\Phi(\lambda, u)=u-T(\lambda, u)$.

LEMMA 5.3. $\Phi$ satisfies:

(a) $\Phi(\lambda, u) \neq 0(\lambda, u) \in I_{\Lambda} \times \partial B\left(0, R_{\Lambda}\right)$,

(b) $\operatorname{deg}\left(\Phi(\lambda, \cdot), B\left(0, R_{\Lambda}\right), 0\right) \neq 0$ for each $\lambda \in I_{\Lambda}$.

Proof. The verification of (a) is straightforward since $T\left(I_{\Lambda} \times \overline{B\left(0, R_{\Lambda}\right)}\right) \subset$ $B\left(0, R_{\Lambda}\right)$.

To prove (b) set $R=R_{\Lambda}$, take $\lambda \in I_{\Lambda}$ and consider the homotopy

$$
\Psi_{\lambda}(t, u)=u-t T(\lambda, u), \quad(t, u) \in[0,1] \times \overline{B(0, R)} .
$$

It follows that $0 \notin \Psi_{\lambda}(I \times \partial B(0, R))$. By the invariance under homotopy property of the Leray-Schauder degree

$$
\operatorname{deg}\left(\Psi_{\lambda}(t, \cdot), B(0, R), 0\right)=\operatorname{deg}\left(\Psi_{\lambda}(0, \cdot), B(0, R), 0\right)=1, \quad t \in[0,1] .
$$

Setting $\Phi(\lambda, u)=u-T(\lambda, u),(\lambda, u) \in I_{\Lambda} \times \overline{B(0, R)}$, we also have

$$
\operatorname{deg}(\Phi(\lambda, \cdot), B(0, R), 0)=1, \quad \lambda \in I_{\Lambda} .
$$

Set $\mathcal{S}_{\Lambda}=\left\{(\lambda, u) \in I_{\Lambda} \times \overline{B(0, R)} \mid \Phi(\lambda, u)=0\right\} \subset \mathcal{G}_{\Lambda}$. By the Leray-Schauder Continuation theorem, there is a connected component $\Sigma_{\Lambda} \subset \mathcal{S}_{\Lambda}$ such that

$$
\Sigma_{\Lambda} \cap\left(\left\{\lambda_{*}\right\} \times \overline{B(0, R)}\right) \neq \emptyset \quad \text { and } \quad \Sigma_{\Lambda} \cap(\{\Lambda\} \times \overline{B(0, R)}) \neq \emptyset .
$$

We point out that $\mathcal{S}_{\Lambda}$ is the solution set of the auxiliary problem

$$
\begin{cases}-\Delta_{p} u=\lambda f_{\Lambda}(u) & \text { in } \Omega, \\ u=0 & \text { on } \partial \Omega,\end{cases}
$$

and, since $\Sigma_{\Lambda} \subset \mathcal{S}_{\Lambda} \subset \mathcal{G}_{\Lambda}$, it follows using the definition of $f_{\Lambda}$ that

$$
\begin{cases}-\Delta_{p} u=\lambda f(u) & \text { in } \Omega, \\ u=0 & \text { on } \partial \Omega,\end{cases}
$$

for $(\lambda, u) \in \Sigma_{\Lambda}$, showing that $\Sigma_{\Lambda} \subset \mathcal{S}$. This ends the proof of Theorem 5.2.

\section{Proof of Theorem 1.3}

Consider $\Lambda$ as introduced in Section 5 and take a sequence $\left\{\Lambda_{n}\right\}$ such that $\lambda_{0}<\Lambda_{1}<\Lambda_{2}<\ldots$ with $\Lambda_{n} \rightarrow \infty$. Set $\beta_{n}=\Lambda_{n}$ and take a sequence $\left\{\alpha_{n}\right\} \subset \mathbb{R}$ such that $\alpha_{n} \rightarrow-\infty$ and $\ldots<\alpha_{n}<\ldots<\alpha_{1}<\lambda_{0}$. 
Following the notations of Section 4, consider the sequence of intervals $I_{n}=$ $\left[\lambda_{0}, \Lambda_{n}\right]$. Set $M=C(\bar{\Omega})$ and let

$$
\mathcal{G}_{\Lambda_{n}}:=\left\{(\lambda, u) \in I_{n} \times \bar{B}_{R_{n}} \mid \underline{u} \leq u \leq \bar{u}, u=0 \text { on } \partial \Omega\right\},
$$

where $R_{n}=R_{\Lambda_{n}}$. Consider the sequence of compact operators

$$
T_{n}:\left[\lambda_{0}, \Lambda_{n}\right] \times \bar{B}_{R_{n}} \rightarrow \bar{B}_{R_{n}}
$$

defined by

$$
\left.T_{n}(\lambda, u)=S\left(\lambda F_{\Lambda_{n}}(u)\right)\right) \quad \text { if } \lambda_{0} \leq \lambda \leq \Lambda_{n}, u \in \bar{B}_{R_{n}} .
$$

Next consider the extension of $T_{n}$, namely $\widetilde{T}_{n}: \mathbb{R} \times \bar{B}_{R_{n}} \rightarrow \bar{B}_{R_{n}}$, defined by

$$
\widetilde{T}_{n}(\lambda, u)= \begin{cases}T_{n}\left(\lambda_{0}, u\right) & \text { if } \lambda \leq \lambda_{0} \\ T_{n}(\lambda, u) & \text { if } \lambda_{0} \leq \lambda \leq \Lambda_{n} \\ T_{n}\left(\Lambda_{n}, u\right) & \text { if } \lambda \geq \Lambda_{n}\end{cases}
$$

Notice that $\widetilde{T}_{n}$ is continuous and compact.

Applying Theorem 5.1 to $\widetilde{T}_{n}:\left[\alpha_{n}, \beta_{n}\right] \times \bar{B}_{R_{n}} \rightarrow \bar{B}_{R_{n}}$, we get a compact connected component $\Sigma_{n}^{*}$ of $\mathcal{S}_{n}=\left\{(\lambda, u) \in\left[\alpha_{n}, \beta_{n}\right] \times \bar{B}_{R_{n}} \mid \Phi_{n}(\lambda, u)=0\right\}$, where

$$
\Phi_{n}(\lambda, u)=u-\widetilde{T}_{n}(\lambda, u) .
$$

Notice that $\Sigma_{n}^{*}$ is also a connected subset of $\mathbb{R} \times M$. By Theorem 1.5 , there is a connected component $\Sigma^{*}$ of $\varlimsup \Sigma_{n}^{*}$ such that

$$
\Sigma^{*} \cap(\{\lambda\} \times M) \neq \emptyset \text { for each } \lambda \in \mathbb{R} .
$$

Set $\Sigma=\left(\left[\lambda_{*}, \infty\right) \times M\right) \cap \Sigma^{*}$. Then $\Sigma \subset \mathbb{R} \times M$ is connected and

$$
\Sigma \cap(\{\lambda\} \times M) \neq \emptyset, \quad \lambda_{0} \leq \lambda<\infty .
$$

We claim that $\Sigma \subset \mathcal{S}$. Indeed, at first notice that

$$
\left.\widetilde{T}_{n+1}\right|_{\left(\left[\lambda_{0}, \Lambda_{n}\right] \times \bar{B}_{R_{n}}\right)}=\left.\widetilde{T}_{n}\right|_{\left(\left[\lambda_{0}, \Lambda_{n}\right] \times \bar{B}_{R_{n}}\right)}=T_{n} .
$$

If $(\lambda, u) \in \Sigma$ with $\lambda>\lambda_{0}$, there is a sequence $\left(\lambda_{n_{i}}, u_{n_{i}}\right) \in \bigcup \Sigma_{n}^{*}$ with $\left(\lambda_{n_{i}}, u_{n_{i}}\right) \in$ $\Sigma_{n_{i}}^{*}$ such that $\lambda_{n_{i}} \rightarrow \lambda$ and $u_{n_{i}} \rightarrow u$, as $n_{i} \rightarrow \infty$. Then $u \in B_{R_{N}}$ for some integer $N>1$.

We can assume that $\left(\lambda_{n_{i}}, u_{n_{i}}\right) \in\left[\lambda_{0}, \Lambda_{N}\right] \times B_{R_{N}}$. On the other hand, by (6.1),

$$
u_{n_{i}}=T_{n_{i}}\left(\lambda_{n_{i}}, u_{n_{i}}\right)=T_{N}\left(\lambda_{n_{i}}, u_{n_{i}}\right) .
$$

Passing to the limit we get $u=T_{N}(\lambda, u)$ which shows that $(\lambda, u) \in \Sigma_{N}$ and so

$$
(\lambda, u) \in \mathcal{S}:=\left\{(\lambda, u) \in(0, \infty) \times C(\bar{\Omega}) \mid u \text { is a solution of }(\mathrm{P})_{\lambda}\right\} .
$$

This ends the proof of Theorem 1.3. 


\section{Appendix A}

In this section we present proofs of Lemma 2.4, Corollary 2.6 and recall some results referred to in the paper. We begin with the Browder-Minty theorem, (cf. Deimling [6]). Let $X$ be a real reflexive Banach space with dual space $X^{*}$. A map $F: X \rightarrow X^{*}$ is monotone if

$$
\langle F x-F y, x-y\rangle \geq 0, \quad x, y \in X,
$$

$F$ is hemicontinuous if

$$
F(x+t y) \stackrel{*}{\rightarrow} F x \quad \text { as } t \rightarrow 0,
$$

and $F$ is coercive if

$$
\frac{\langle F x, x\rangle}{|x|} \rightarrow \infty \quad \text { as }|x| \rightarrow \infty .
$$

Theorem 1.1. Let $X$ be a real reflexive Banach space and let $F: X \rightarrow X^{*}$ be a monotone, hemicontinous and coercive operator. Then $F(X)=X^{*}$. Moreover, if $F$ is strictly monotone then it is a homeomorphism.

The inequality below, (cf. [22], [20]), is very useful when dealing with the $p$-Laplacian.

Lemma 1.2. Let $p>1$. Then there is a constant $C_{p}>0$ such that

$$
\left(|x|^{p-2} x-|y|^{p-2} y, x-y\right) \geq \begin{cases}C_{p}|x-y|^{p} & \text { if } p \geq 2, \\ C_{p} \frac{|x-y|^{p}}{(1+|x|+|y|)^{2-p}} & \text { if } \quad p \leq 2,\end{cases}
$$

where $x, y \in \mathbb{R}^{N}$ and $(\cdot, \cdot)$ is the usual inner product of $\mathbb{R}^{N}$.

Recall the Hardy inequality (cf. Brézis [3]).

TheOREM 1.3. There is a positive constant $C$ such that

$$
\int_{\Omega}\left|\frac{u}{d}\right|^{\beta} d x \leq C \int_{\Omega}|\nabla u|^{p}, \quad u \in W_{0}^{1, p}(\Omega) .
$$

Proof of Lemma 2.4. By the Hölder inequality,

$$
\int_{\Omega}|\nabla u|^{p-1}|\nabla v| d x \leq\|u\|_{1, p^{\prime}}\|v\|_{1, p},
$$

where $1 / p+1 / p^{\prime}=1$, and so the expression

$$
\left\langle-\Delta_{p} u, v\right\rangle:=\int_{\Omega}|\nabla u|^{p-2} \nabla u \cdot \nabla v d x, \quad u, v \in W_{0}^{1, p}(\Omega),
$$

defines a continuous, bounded (nonlinear) operator, namely

$$
\Delta_{p}: W_{0}^{1, p}(\Omega) \rightarrow W^{-1, p^{\prime}}(\Omega), \quad u \mapsto \Delta_{p} u .
$$

By (A.1),$-\Delta_{p}$ it is strictly monotone and coercive, that is

$$
\left\langle-\Delta_{p} u-\left(-\Delta_{p} v\right), u-v\right\rangle>0, \quad u, v \in W_{0}^{1, p}(\Omega), u \neq v
$$


and

$$
\frac{\left\langle-\Delta_{p} u, u\right\rangle}{\|u\|_{1, p}} \stackrel{\|u\|_{1, p} \rightarrow \infty}{\longrightarrow} \infty .
$$

By the Browder-Minty theorem, $\Delta_{p}: W_{0}^{1, p}(\Omega) \rightarrow W^{-1, p^{\prime}}(\Omega)$ is a homeomorphism.

Consider

$$
F_{g}(u)=\int_{\Omega} g u d x, \quad u \in W_{0}^{1, p}(\Omega) .
$$

Claim. $F_{g} \in W^{-1, p^{\prime}}(\Omega)$.

Assume for a while the claim has been proved. Since $-\Delta_{p}: W_{0}^{1, p}(\Omega) \rightarrow$ $W^{-1, p^{\prime}}(\Omega)$ is a homeomorphism, there is only $u \in W_{0}^{1, p}(\Omega)$ such that $-\Delta_{p} u=$ $F_{g}$, that is

$$
\left\langle-\Delta_{p} u, v\right\rangle=\int_{\Omega} g v d x, \quad v \in W_{0}^{1, p}(\Omega)
$$

Verification of Claim. Let $V$ be an open neighborhood of $\partial \Omega$ such that $0<d(x)<1$ for $x \in V$ so that

$$
1<\frac{1}{d(x)^{\beta}}<\frac{1}{d(x)}, \quad x \in V .
$$

Now, if $v \in W_{0}^{1, p}(\Omega)$ we have

$$
\left|F_{g}(v)\right| \leq \int_{\Omega}|g||v| d x=\int_{V^{c}}|g||v| d x+\int_{V}|g||v| d x \leq C\|v\|_{1, p}+\int_{\Omega}\left|\frac{v}{d}\right| d x .
$$

Applying the Hardy inequality in the last term above, we get to

$$
\left|F_{g}(v)\right| \leq C\|v\|_{1, p},
$$

showing that $F_{g} \in W^{-1, p^{\prime}}(\Omega)$, proving the claim.

Regularity of $u$. At first we treat the case $p=2$. By [5], there is a solution $v$ of

$$
\begin{cases}-\Delta v=1 / v^{\beta} & \text { in } \Omega, \\ v>0 & \text { in } \Omega, \\ v=0 & \text { on } \partial \Omega,\end{cases}
$$

which belongs to $C^{1}(\bar{\Omega})$ and by the Hopf theorem $\partial v / \partial \nu<0$ on $\partial \Omega$. Since also $d \in C^{1}(\bar{\Omega})$ and $\partial d / \partial \nu<0$ on $\partial \Omega$ there a constant $C>0$ such that $v \leq C d$ in $\Omega$. Moreover, $-\Delta v=1 / v^{\beta} \geq C / d^{\beta}$. Consider the problem

$$
\begin{cases}-\Delta \widetilde{u}=|g| & \text { in } \Omega, \\ \widetilde{u}=0 & \text { on } \partial \Omega .\end{cases}
$$

By [11, theorem B.1], $\widetilde{u} \in C^{1, \alpha}(\bar{\Omega})$ and $\|\widetilde{u}\|_{C^{1, \alpha}(\bar{\Omega})} \leq M_{0}$ for some positive constant $M_{0}$. By the Maximum Principle, $\widetilde{u} \leq v \leq C d$ in $\Omega$. 
Setting $\bar{u}=u+\widetilde{u}$ we get $-\Delta \bar{u}=g+|g| \geq 0$ in $\Omega$ and by the arguments above, $\bar{u} \leq C d$ in $\Omega$. Thus, as a consequence of [11, Theorem B.1], there are $\alpha \in(0,1)$ and $M_{0}>0$ such that

$$
\bar{u}, \widetilde{u} \in C^{1, \alpha}(\bar{\Omega}) \quad \text { and } \quad\|\bar{u}\|_{C^{1, \alpha}(\bar{\Omega})},\|\widetilde{u}\|_{C^{1, \alpha}(\bar{\Omega})} \leq M_{0},
$$

ending the proof of Lemma 2.4 in the case $p=2$.

In what follows we treat the case $p>1$. Let $u$ be a solution of (2.3). It follows that

$$
-\Delta_{p} u=g \leq \frac{C}{d^{\beta}} \quad \text { and } \quad-\Delta_{p}(-u)=(-1)^{p-1} g \leq \frac{C}{d^{\beta}} .
$$

By Lemma 2.2, the problem

$$
\begin{cases}-\Delta_{p} v=C / v^{\beta} & \text { in } \Omega, \\ v=0 & \text { on } \partial \Omega\end{cases}
$$

admits only a positive solution $v \in W_{0}^{1, p}(\Omega) \cap C^{1, \alpha}(\bar{\Omega})$ for some $\alpha \in(0,1)$ with $v \leq C d$ in $\Omega$. Hence,

Therefore,

$$
-\Delta_{p}(v)=\frac{C}{v^{\beta}} \geq \frac{1}{d^{\beta}} \quad \text { in } \Omega .
$$

$$
-\Delta_{p}|u| \leq \frac{C}{d^{\beta}} \leq-\Delta_{p} v .
$$

By the Weak Comparison Principle, $|u| \leq v \leq C d$ in $\Omega$, showing that $u \in L^{\infty}(\Omega)$. Pick $w \in C^{1, \alpha}(\bar{\Omega})$ such that

$$
-\Delta w=g \quad \text { in } \Omega, \quad w=0 \quad \text { on } \partial \Omega .
$$

We have

$$
\operatorname{div}\left(|\nabla u|^{p-2} \nabla u-\nabla w\right)=0 \quad \text { in } \Omega
$$

in the weak sense. By Lieberman [17, Theorem 1] the proof of Lemma 2.4 ends.

Proof of Corollary 2.6. Existence of $u_{\varepsilon}$ follows directly by Lemma 2.4. Moreover, there are $M>0$ and $\alpha \in(0,1)$ such that

$$
\|u\|_{C^{1, \alpha}(\bar{\Omega})},\left\|u_{\varepsilon}\right\|_{C^{1, \alpha}(\bar{\Omega})}<M .
$$

By Vázquez [25, Theorem 5], $\partial u / \partial \nu<0$ on $\partial \Omega$ and recalling that $d \in C^{1}(\bar{\Omega})$ and $\partial d / \partial \nu<0$ on $\partial \Omega$ it follows that

$$
u \geq C d \quad \text { in } \Omega .
$$

Multiplying the equation

$$
-\Delta_{p} u-\left(-\Delta_{p} u_{\varepsilon}\right)=g-\left(h \chi_{[d(x)>\varepsilon]}+\widetilde{g} \chi_{[d(x)<\varepsilon]}\right)
$$

by $u-u_{\varepsilon}$ and integrating we have

$$
\int_{\Omega}\left(|\nabla u|^{p-2} \nabla u-\left|\nabla u_{\varepsilon}\right|^{p-2} \nabla u_{\varepsilon}\right) \cdot \nabla\left(u-u_{\varepsilon}\right) d x \leq 2 M \int_{d(x)<\varepsilon}|g-\widetilde{g}| d x .
$$


Using Lemma 1.2, we infer that $\left\|u-u_{\varepsilon}\right\|_{1, p} \rightarrow 0$ as $\varepsilon \rightarrow 0$. By the compact embedding $C^{1, \alpha}(\bar{\Omega}) \hookrightarrow C^{1}(\bar{\Omega})$, it follows that

$$
\left\|u-u_{\varepsilon}\right\|_{C^{1}(\bar{\Omega})} \leq \frac{C}{2} d
$$

and, using (A.4),

$$
u_{\varepsilon} \geq u-\frac{C}{2} d \geq u-\frac{u}{2}=\frac{u}{2} .
$$

\section{REFERENCES}

[1] A. AnAne, Simplicité et isolation de la primiére valeur propre du p-Laplacien avec poids, C.R. Acad. Sci. Paris Sér. I (1987), 725-728.

[2] L. Boccardo, F. Murat and J.P. Puel, Résultats d'existence pour certains problémes elliptiques quasilinéaires, Ann. Sc. Norm. Super. Pisa Cl. Sci. 2 (1984), 213-235.

[3] H. BrÉzIS, Functional Analysis, Sobolev Spaces and partial differential equations, Springer-Verlag, Berlin, (2011)

[4] S. Carl and K. Perera, Generalized solutions of singular p-Laplacian problems in $\mathbb{R}^{N}$, Nonlinear Stud. 18 (2011), 113-124.

[5] M.G. Crandall, P.H. Rabinowitz and L. Tartar, On a Dirichlet problem with a singular nonlinearity, Comm. Partial Differential Equations 2 (1977), 193-222.

[6] K. Deimling, Nonlinear Functional Analysis, Springer-Verlag, Berlin, 1985.

[7] J.I. Diaz, J. Hernandez and F.J. Mancebo, Branches of positive and free boundary solutions for some singular quasilinear elliptic problem, J. Math. Anal. Appl. 352 (2009) 449-474.

[8] J.I. Diaz, J.M. Morel And L. Oswald, An elliptic equation with singular nonlinearity, Comm. Partial Differential Equations 12 (1987), 1333-1344.

[9] E. DiBenedetto, $C^{1+\alpha}$-local regularity of weak solutions of degenerate elliptic equations, Nonlinear Anal. 7 (1983), 827-850.

[10] M. Ghergu And V. Radulescu, Sublinear singular elliptic problems with two parameters, J. Differential Equations 195 (2003), 520-536.

[11] J. Giacomoni, I. Schindler And P. TAKaC, Sobolev versus Hölder local minimizers and existence of multiple solutions for a singular quasilinear equation, Ann. Sc. Norm. Super. Pisa Cl. Sci. (5) 6 (2007), 117-158.

[12] D. Gilbarg and N.S. Trudinger, Elliptic Partial Differential Equations of Second Order, Springer-Verlag, New York, 1983.

[13] J.V. Goncalves, M.C. Rezende and C.A. Santos, Positive solutions for a mixed and singular quasilinear problem, Nonlinear Anal. 74 (2011), 132-140.

[14] D.D. HAI, Singular boundary value problems for the p-Laplacian, Nonlinear Anal. 73 (2010), 2876-2881.

[15] $ـ$ On a class of singular p-Laplacian boundary value problems, J. Math. Anal. Appl. 383 (2011), 619-626.

[16] A.C. Lazer and P.J. McKenna, On a singular nonlinear elliptic boundary value problem, Proc. Amer. Math. Soc. 111 (1991), 721-730.

[17] G.M. Liebermann, Boundary regularity for solutions of degenerate elliptic equations, Nonlinear Anal. 12 (1988), 1203-1219.

[18] N.H. Loc And K. Schmitt, Boundary value problems for singular elliptic equations, Rocky Mountain J. Math. 41 (2011), 555-572. 
[19] A. Mohammed, Positive solutions of the p-Laplace equation with singular nonlinearity, J. Math. Anal. Appl. 352 (2009), 234-245.

[20] I. PerAL, Multiplicity of Solutions for the p-Laplacian, Second School on Nonlinear Functional Analysis and Applications to Differential Equations, Trieste, Italy, 1997.

[21] J. Shi And M. YaO, On a singular nonlinear semilinear elliptic problem, Proc. Roy. Soc. Edinburgh Sect. A 138 (1998), 1389-1401.

[22] J. Simon, Regularité de la solution d'une equation non linéaire dans $\mathbb{R}^{N}$, Lecture Notes in Mathematics, vol. 665, Springer-Verlag, New York, 1978.

[23] J.X. Sun AND F.M. Song, A property of connected components and its applications, Topology Appl. 125 (2002), 553-560.

[24] P. TOLKSDORFF, Regularity for a more general class of quasilinear elliptic equations, J. Differential Equations 51 (1984), 126-150.

[25] J.L. VÁzQuez, A strong maximum principle for some quasilinear elliptic equations, Appl. Math. Optim. 12 (1984), 191-202.

[26] G.T. Whyburn, Topological Analysis, Princeton University Press, Princeton NJ, 1955.

Manuscript received July 2, 2014

accepted February 3, 2015

José V. GonÇALVES

Universidade Federal de Goiás

Instituto de Matemática e Estatística

74001-970 Goiânia, GO, BRAZIL

E-mail address: goncalves.jva@gmail.com

Marcos R. MARcial

Universidade Federal de Ouro Preto

Departamento de Matemática - ICEB

Campus Universitário Morro do Cruzeiro

35400-000 Ouro Preto, MG, BRAZIL

E-mail address: marcosrobertomarcial@yahoo.com.br

Olimpio H. MiYagaki

Universidade Federal de Juiz de Fora

Departamento de Matemática

36036-330 Juiz de Fora, MG, BRAZIL

E-mail address: ohmiyagaki@gmail.com

TMNA : Volume $47-2016-\mathrm{N}^{\mathrm{O}} 1$ 